\title{
Relación entre la motivación académico-personal del estudiante novel en educación y las estrategias de trabajo autónomo
}

\author{
María C. Pegalajar-Palomino \\ Facultad de Humanidades y Ciencias de la Educación, Departamento de Pedagogía, Universidad de Jaén, Campus Las \\ Lagunillas s/n, Jaén - España (correo-e: mcpegala@ujaen.es).
}

Recibido Mar. 12, 2020; Aceptado May. 12, 2020; Versión final Jun. 11, 2020; Publicado Oct. 2020

\begin{abstract}
Resumen
Este trabajo analiza la relación entre la motivación académico-personal del estudiante universitario novel y las estrategias de trabajo autónomo desarrolladas en su proceso de formación inicial. La investigación está basada en una metodología descriptivo-correlacional, utilizándose la encuesta como técnica de recogida de datos mediante cuestionarios validados. La muestra está compuesta por estudiantes de primer curso del grado en Educación Infantil y Primaria de dos universidades españolas. Las estrategias de preparación de exámenes y conceptualización son las más desarrolladas por los estudiantes, quienes acceden a la educación superior por motivos personales y por las condiciones en que realizan dichos estudios. Se perciben correlaciones entre las variables estudiadas, detectándose diferencias significativas en dichas variables según la edad del estudiante y la titularidad de la universidad. Se destaca la importancia de explorar el perfil del estudiante para el desarrollo de un proceso de aprendizaje eficaz y de mayor calidad en educación superior.
\end{abstract}

Palabras clave: estudiante; aprendizaje activo; método de enseñanza; autoaprendizaje; motivación

\section{Relationship between academic-personal motivation of freshman pedagogy students and individual learning strategies}

The present study analyzes the relationship between academic and personal motivation of the novel university student and individual learning strategies developed in her/his initial training process. The research is based on a descriptive-correlational methodology, using the survey as a data collection technique through validated questionnaires. The sample is composed of first year students in the degree of early childhood and primary education from two Spanish universities. The results show that exam preparation and conceptualization strategies are the most developed by the students. Students enter higher education for personal reasons and because of the conditions in which they pursue their studies. Correlations between the variables studied included significant differences according to student's age and university ownership. This study highlights the importance of exploring student profiles for developing an effective and improved quality learning process in higher education. 


\section{INTRODUCCIÓN}

La rapidez con que se están produciendo las actuales transformaciones socioeconómicas, científicas y tecnológicas ha posibilitado un cambio en el carácter de las instituciones educativas. A día de hoy, nuestra sociedad otorga mucha importancia al aprendizaje a lo largo de la vida, proliferando entornos didácticos informales que requieren del desarrollo de habilidades de autorregulación en el estudiante (Beishuizen y Steffens, 2011). Así pues, el aprendizaje autorregulado supone un proceso personal de activación sistemática de variables de tipo cognitivo, afectiva y comportamental dirigido a la consecución de una meta académica (Zimmerman y Schunk, 2011). Desde la Educación Superior se debe potenciar en el estudiante la capacidad de aprender de manera autónoma y con iniciativa personal, despertando su interés por desarrollar un aprendizaje permanente (Marcelo y Rijo, 2019). Según los nuevos modelos educativos, la motivación resulta un factor de primer orden para afrontar un aprendizaje más autónomo con mayor nivel de exigencia y esfuerzo en el estudiante (Romero y Pérez, 2009). Se trata de un proceso cognitivo que incide sobre el papel de los pensamientos del estudiante, sus creencias y emociones y está dirigido hacia un objetivo o meta que la provoca y mantiene (Pintrich y Schunk, 2006).

Así pues, las estrategias de aprendizaje puestas en práctica por el estudiante de manera autónoma le permiten alcanzar un aprendizaje significativo a partir del control sobre las situaciones en que dicho proceso se desarrolla (Yang y Kim, 2014). Dichas estrategias favorecen el desarrollo de la competencia "aprender a aprender" (Gargallo et al. 2020), posibilitando que el estudiante pueda tomar decisiones adecuadas sobre qué y cómo aprender según el contexto y los objetivos de aprendizaje (Gargallo et al., 2016). López-Aguado (2010) sostiene que las estrategias de aprendizaje autónomo en el estudiante universitario implican una serie de variables que incluyen: 1) Ampliación del contenido mediante la búsqueda y producción de materiales, participación en actividades complementarias, etc.; 2) Colaboración en tareas grupales a partir de procesos de aprendizaje cooperativo; 3) Conceptualización y trabajo intelectual sobre el contenido de la asignatura; 4) Planificación y organización temporal del estudio y la programación de las tareas; 5) Preparación de exámenes para la evaluación y 5) Participación del estudiante en el proceso.

Además, la gestión de los recursos motivacionales y afectivos es una de las líneas más sobresalientes de la investigación sobre las estrategias de aprendizaje en el estudiante (Cabanach et al., 2009). Simons y Chen (2009) entienden la motivación académica como la generalización con que los estudiantes se comprometen con la institución educativa y se muestran receptivos hacia el aprendizaje. Boza y Méndez (2013) exponen algunas de las variables con que se relaciona este concepto, siendo éstas: 1) Metas vitales de carácter afectivo, cognitivo, auto asertivo, social o de la tarea; 2) Actitudes hacia el aprendizaje; 3) Motivos personales para el aprendizaje; 4) Condiciones de estudio; 5) Estrategias de aprendizaje y 6) Implicación del alumnado en el estudio.

Resulta de interés investigar acerca de las estrategias de aprendizaje autónomo y la motivación hacia el aprendizaje en el estudiante que accede a la universidad, pues la transición a la Educación Superior supone un proceso gradual y multidimensional que requiere de distintas respuestas de afrontamiento para su posterior éxito académico (Nightingale et al., 2013). En este sentido, López-Cárdenas et al. (2018) subrayan la importancia de indagar sobre las condiciones de partida del estudiante que ingresa en la universidad, con la intención de organizar actuaciones institucionales que permitan una transición exitosa a la Educación Superior. Para ello, el profesorado debe preocuparse por buscar y mantener la calidad de la educación para garantizar unos mejores resultados de aprendizaje en sus estudiantes (Herrera et al., 2018), así como también fomentar su participación y motivación hacia el estudio (De Soto, 2018). La realidad demuestra cómo los estudiantes universitarios noveles necesitan capacitarse para el desarrollo de la competencia de "aprender a aprender" (Trautwein y Bosse, 2017; Wibrowski et al., 2016). Así pues, y a pesar de dominar distintas estrategias de aprendizaje, el alumnado universitario tiene dificultades para emplearlas, siendo éste uno de sus principales problemas a nivel académico (Başbaği y Yilmaz, 2015).

La cantidad y calidad de las estrategias que el estudiante utiliza en su aprendizaje son uno de los factores que identifican a los buenos estudiantes (García-Valcárcel y Tejedor, 2017), con excelente rendimiento académico (Soares et al., 2009; Yip, 2012). Los valores más empleados por los estudiantes dentro de los factores de motivación son las metas intrínsecas, el valor de la tarea y la autoeficacia, entendida esta última como la creencia que tiene el estudiante sobre su capacidad para desempeñar las actividades académicas que se le demandan (Bandura, 1997). Los estudiantes con elevada autoeficacia tienden a obtener altas calificaciones académicas (Lee et al., 2014), mejor comportamiento y participación en el aula (Galyon et al., 2012) e interés por el aprendizaje (Weber y Ruch, 2012). Por su parte, las estrategias de aprendizaje más utilizadas son las de elaboración, organización del proceso y metacognición (Garrote et al., 2016; Roys y Pérez, 2018). Sin embargo, Castro y Miranda (2019) describen cómo el alumnado se motiva a estudiar si responde a sus necesidades según determinados factores externos, como pueda ser: relación social, competencia o autonomía. 
Tomando como referencia el desarrollo de estrategias de aprendizaje y las variables motivacionales hacia el estudio en alumnado universitario según su edad, cabe destacar la escasez de investigaciones al respecto. No obstante, Gargallo y Suárez (2014) y Tasoobshirazi y Carr (2009) sostienen cómo el alumnado de curso superiores y, por consiguiente, de más edad, hace un mayor uso de estrategias de aprendizaje eficaces, utilizando un enfoque de aprendizaje más profundo (García-Berbén, 2005; Hernández et al., 2002). Por el contrario, otros autores como Lynch (2008) describen cómo el mantenimiento del esfuerzo disminuye en el estudiante de cursos superiores.

Por otro lado, y según la titularidad de la institución de Educación Superior, Maquilón y Hernández (2011) describen cómo el hecho de estudiar en un centro educativo de carácter público o privado está relacionado con el uso predominante de un determinado enfoque de aprendizaje, aunque influyen otra serie de variables. Sin embargo, las investigaciones de Castro et al. (2019) y Coterón et al. (2013) revelan una mayor orientación hacia el ego en alumnado que estudian en centros privados. De este modo, se necesita determinar cuáles son los métodos idóneos para generar un aprendizaje sólido, útil y transferible en el estudiante de Educación Superior (Powell y Robson, 2014), teniendo en cuenta sus características de aprendizaje y aunando las estrategias de enseñanza-aprendizaje (Garrote et al. 2016).

Para ello, este estudio pretende analizar la relación entre las motivaciones académico-personales y el desarrollo de estrategias de aprendizaje autónomo puestas en práctica por el estudiante universitario novel de Educación. Parte de un trabajo previo basado en el análisis de las estrategias de trabajo autónomo del estudiante novel adscrito al Grado en Educación, con la intención de acercar al profesorado universitario al conocimiento de las estrategias y habilidades de que dispone el estudiante para adaptar el proceso de enseñanza-aprendizaje (Pegalajar, 2020). Por ello, el interés de este trabajo reside en la necesidad de conocer la correspondencia entre ambos constructos para evitar el fracaso académico del estudiante que accede al sistema universitario. El nuevo modelo educativo en Educación Superior sitúa al estudiante como agente principal en el proceso de aprendizaje, siendo el profesor un agente vinculado a la orientación y el asesoramiento, por lo que resulta crucial conocer y analizar dichos elementos para garantizar una mejor calidad de la Educación Superior.

No obstante, y de modo más concreto, se persiguen los siguientes objetivos específicos: 1) Identificar las estrategias más habituales de trabajo autónomo en estudiantes de los Grados en Educación Infantil y Primaria; 2) Examinar las metas, actitudes, motivos, condiciones, estrategias e implicación hacia el estudio del alumnado de los Grados en Educación Infantil y Primaria; 3) Conocer la existencia de relaciones estadísticamente significativas entre la motivación académico-personal del estudiante universitario novel en Educación y la puesta en práctica de estrategias de trabajo autónomo; 4) Comprobar la existencia de diferencias estadísticamente significativas en las percepciones de los estudiantes sobre su motivación académica y el desarrollo de estrategias de trabajo autónomo según la edad del estudiante y la titularidad de la Universidad en que realizan sus estudios.

\section{METODOLOGÍA}

Este estudio presenta un carácter cuantitativo, pues se trata de una investigación descriptivo-correlacional que pretende conocer el grado de relación que existe entre dos variables consideradas de interés. Desde la Educación Superior, se debe examinar el punto de partida del estudiante para adaptar el proceso de enseñanza-aprendizaje a sus características y necesidades individuales.

\section{Participantes}

La población objeto de estudio de esta investigación está compuesta por estudiantes de primer curso matriculados durante el curso académico 2018/19 en los Grados en Educación Infantil y Educación Primaria de la Universidad de Jaén (de titularidad pública) y la Universidad Católica de Murcia (de titularidad privada). Según datos aportados por las secretarías académicas de ambas universidades, la cifra asciende a un total de 853 estudiantes de nuevo ingreso para ambas titulaciones. La muestra final del estudio supone un total de 772 estudiantes, utilizándose un muestreo no probabilístico de tipo incidental, de tal forma que han quedado adscritos a la investigación todos los estudiantes que han contestado al cuestionario facilitado por el profesorado al comienzo del curso. Para el cálculo de la muestra participante se ha utilizado la fórmula para poblaciones con menos de 100.000 sujetos, con un nivel de confianza del $95 \%$ y un error de estimación máximo de 4\%. Además, la prueba Kolmogorov-Smirnov ha permitido comprobar que la muestra sigue una distribución normal; el análisis realizado ha revelado valores superiores a $p \leq, 05$, siendo el caso mayor $z=, 568 p \leq, 904$. La Tabla 1 describe las características sociodemográficas de la muestra. 
Tabla 1: Características de la muestra

\begin{tabular}{|l|l|l|l|}
\hline Variable & Hombre & $\mathrm{N}$ & $\%$ \\
\hline \multirow{3}{*}{ Género } & Mujer & 335 & 43,4 \\
\hline \multirow{4}{*}{ Edad } & Menos de 20 años & 437 & 56,6 \\
\cline { 2 - 4 } & De 21 a 30 años & 410 & 53,1 \\
\cline { 2 - 4 } & Más de 31 años & 256 & 33,2 \\
\hline \multirow{3}{*}{ Gniversidad } & Pública & 106 & 13,7 \\
\cline { 2 - 4 } & Privada & 476 & 61,6 \\
\hline \multirow{3}{*}{ Acceso } & Educación Infantil & 296 & 38,4 \\
\cline { 2 - 4 } & Educación Primaria & 435 & 56,3 \\
\cline { 2 - 4 } & Bachillerato Humanidades y Ciencias Sociales & 337 & 43,7 \\
\cline { 2 - 4 } & Bachillerato Ciencia y Tecnología & 449 & 58,2 \\
\cline { 2 - 4 } & Ciclos Formativos de Grado Superior (Animación Sociocultural, Integración & 94 & 12,2 \\
\cline { 2 - 4 } & Social, Educación Infantil, Lengua de signos, etc.) & 180 & 23,4 \\
\cline { 2 - 4 } & Otras opciones (pruebas de acceso para mayores de 25, mayores de 45, etc.) & 49 & 6,2 \\
\hline
\end{tabular}

\section{Instrumentos}

El "Cuestionario de Estrategias de Trabajo Autónomo" (López-Aguado, 2010) consiste en una escala tipo Likert compuesta por 45 ítems con cinco opciones de respuesta (siendo 1=nunca; 2=pocas veces; $3=$ algunas veces; $4=$ muchas veces y $5=$ siempre) y dividida en 6 subescalas, las cuáles abordan el análisis de las distintas variables, siendo éstas: 1) Ampliación a partir de la búsqueda de materiales y la realización de actividades complementarias a las propuestas por el profesor; 2) Colaboración e implicación del estudiante en actividades grupales y de trabajo colaborativo con sus iguales; 3) Conceptualización y trabajo intelectual sobre el contenido de las materias (elaboración de esquemas, resúmenes, mapas conceptuales, etc.); 4) Planificación de tiempos, ejecución de tareas y evaluación de los procedimientos de aprendizaje; 5) Preparación de exámenes, a partir del estudio y la selección de los aspectos fundamentales de cada tema y la realización de actividades de repaso y 6) Participación del alumnado en el proceso de enseñanzaaprendizaje mediante la asistencia a clase, aclaración de dudas, participación en tutorías, etc.

En su versión original, dicho instrumento posee unas cualidades psicométricas favorables. Cabe destacar la adecuada distribución para el análisis del poder discriminativo de los ítems, así como el elevado coeficiente de fiabilidad (,898). Por su parte, el índice Kaisar-Meyer-Olkin (KMO=,845) informa de una correcta adecuación muestral, existiendo interrelaciones significativas entre las variables que alertan de la adecuación de los datos para el modelo de análisis factorial.

La "Escala de autopercepción de la motivación académica y personal” (Boza y Méndez, 2013), se centra en el análisis de autopercepciones en el alumnado sobre su nivel de motivación e interés hacia los estudios que cursa. Consiste en una escala tipo Likert (con opciones de respuesta entre $1=$ nada de acuerdo y $5=$ totalmente de acuerdo), compuesta por 84 ítems, distribuidos en cinco dimensiones tales como: 1) Metas vitales, las cuales incluyen variables de carácter afectivo, cognitivo, autoasertivo, social y de tarea; 2) Actitudes ante el aprendizaje, vinculado a la orientación al éxito, valor de la tarea, atribución de logro interna y/o externa, resignación ante el fracaso, evitación de fracaso y sobreesfuerzo; 3) Motivos personales, la cual incluye personales propios (de aprendizaje, expectativas personales, familia, independencia, responsabilidad, bienestar interior, superación, importancia del saber, vocación, maduración, creatividad y vida organizada), satisfacción con la tarea (amistad, diversión) y de trabajo/profesión (exigencia de estudios, trabajo deseado); 4) Condiciones e implicación en el estudio para el análisis de la competencia, planificación, esfuerzo, participación, dedicación, lugar, responsabilidad, autonomía y persistencia y 5) Estrategias de aprendizaje, basada en el análisis de los conocimientos previos de que dispone el estudiante, uso de fuentes adecuadas, recursos diferentes, contenidos relevantes, lecturas globales, síntesis, anotaciones, etc.

Los resultados arrojados para la validación de la escala, en su versión original, permiten concluir que se trata de un instrumento válido y fiable. El análisis de fiabilidad global desvela unos valores altos (,915), así como los propios de cada bloque (cuyos valores oscilan entre ,676 y ,855). Por su parte, el análisis de la validez de constructo, realizado a partir de un análisis factorial para cada uno de los factores, comprueba que la matriz de correlaciones no es una matriz de identidad. 


\section{Procedimiento}

El procedimiento para la recogida de datos ha sido similar en ambos centros universitarios, con la intención de no alterar los resultados. El acceso a la muestra se ha desarrollado a partir de una asignatura de carácter obligatoria incluida en el plan de estudios de cada uno de los Grados en ambas instituciones educativas. Previo a la aplicación de los instrumentos, se ha contado con el visto bueno de los coordinadores de las asignaturas y el profesorado implicado en su docencia, a quiénes se les ha explicado en qué consiste la investigación y se les ha solicitado el permiso necesario para llevarla a cabo.

Finalmente, se ha informado al alumnado del objetivo de la investigación asegurándole, en todo momento, el anonimato y la confidencialidad de los datos recogidos para así facilitar su participación. Además, los estudiantes han recibido las normas y explicaciones necesarias para su adecuada cumplimentación. Además, y con la intención de que la metodología docente no afectase a los resultados obtenidos, se ha tenido especial atención y cuidado con el procedimiento para la recogida de datos, siendo facilitados los cuestionarios a los estudiantes de manera individual justo al comienzo de las clases. En total, los dos cuestionarios incluyen 129 ítems, por lo que se ha preferido pasarlos a los estudiantes en dos sesiones distintas, para evitar que la variable cansancio y motivación ante la tarea, pueda modificar los resultados.

\section{Análisis de datos}

El análisis de datos se ha realizado a través del programa Statistical Package for the Social Sciences (versión 22 para Windows), llevándose a cabo un análisis descriptivo para cada una de las variables objeto de estudio de esta investigación y un análisis de correlación para conocer su grado de relación, obtenido a partir del coeficiente de correlación de Pearson. Además, se ha analizado la existencia o no de diferencias significativas a nivel estadístico según variables cuantitativas (edad del estudiante) y cualitativas (titularidad de la universidad: pública o privada). Para el primer caso, se ha utilizado un análisis de varianza "ANOVA"; la prueba Tukey realizada a posteriori ha determinado entre qué grupos se aprecian dichas diferencias, mientras que en el caso de la variable cualitativa se ha utilizado la prueba $t$ de Student de comparación de medias. En el apartado de resultados, se exponen los datos obtenidos en las distintas tablas resaltándose aquellos que han obtenido valores significativos mediante el símbolo *.

\section{RESULTADOS}

Tal y como se muestra en la Tabla 2, el análisis descriptivo de las variables motivacionales determina cómo las mejores puntuaciones corresponden a los motivos personales del alumnado para el estudio $(M=4,11$; $\mathrm{SD}=, 43$ ), al considerar interesante la experiencia de formarse en la universidad, responder a sus expectativas personales, adquirir conocimientos, satisfacer el deseo de superación personal, poder optar en un futuro a mejores condiciones en el mercado de trabajo, tener una vida organizada y gozar de independencia y libertad.

Tabla 2: Medias, desviaciones típicas y correlaciones entre las variables estudiadas

\begin{tabular}{|c|c|c|c|c|c|c|c|c|c|c|c|}
\hline & 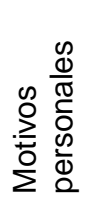 & 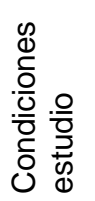 & 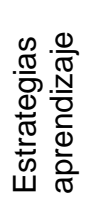 & $\begin{array}{l}0 \\
\frac{0}{\pi} \\
\frac{\pi}{3} \\
0 \\
\frac{\pi}{0} \\
\sum \\
\sum\end{array}$ & 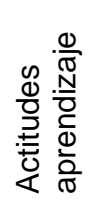 & 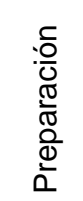 & 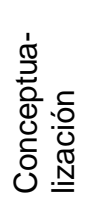 & $\begin{array}{l}\frac{\overline{0}}{0} \\
\frac{0}{0} \\
\frac{0}{0} \\
\frac{0}{0} \\
\frac{0}{0} \\
0\end{array}$ & 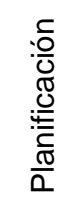 & $\begin{array}{l}\frac{}{0} \\
\frac{0}{0} \\
\frac{0}{0} \\
\frac{0}{0} \\
0\end{array}$ & $\begin{array}{l}: \frac{.0}{0} \\
\frac{.0}{\bar{O}} \\
\frac{0}{\underline{O}} \\
\frac{\varepsilon}{4}\end{array}$ \\
\hline Motivos personales & 1 & ,46* &, $44^{\star}$ & ,60* & ,33* & ,25 & ,13 &,- 14 & ,22 & ,18 & ,05 \\
\hline Condiciones estudio & & 1 &, $53^{\star}$ & $27^{*}$ &, $17^{*}$ &, $33^{*}$ & ,11 &,- 21 & ,15 & ,13 & ,13 \\
\hline $\begin{array}{l}\text { Estrategias } \\
\text { aprendizaje }\end{array}$ & & & 1 &, $42^{*}$ & $18^{*}$ & ,30 & ,10 &,- 06 & ,23 & $34^{*}$ & , 18 \\
\hline Metas vitales & & & & 1 & $46^{*}$ & ,26 & ,21 & ,07 &, $50^{*}$ & ,02 & ,20 \\
\hline Actitudes aprendizaje & & & & & 1 &, $38^{*}$ & $30^{*}$ & ,01 & $40^{*}$ & , 17 & ,25 \\
\hline Preparación & & & & & & 1 & $62^{*}$ & $45^{*}$ &, $54^{*}$ & $40^{*}$ &, $51^{*}$ \\
\hline Conceptualización & & & & & & & 1 & $36^{*}$ & $45^{*}$ & $32^{*}$ &, $51^{*}$ \\
\hline Participación & & & & & & & & 1 &, $36^{*}$ & $41^{*}$ &, $50^{*}$ \\
\hline Planificación & & & & & & & & & 1 & $35^{\star}$ &, $50^{*}$ \\
\hline Colaboración & & & & & & & & & & 1 &, $54^{*}$ \\
\hline Ampliación & & & & & & & & & & & 1 \\
\hline M. & 4,11 & 3,73 & 3,53 & 3,46 & 3,03 & 3,78 & 3,66 & 3,47 & 3,12 & 3,06 & 2,89 \\
\hline D.T. & ,43 & ,53 & 67 &, 52 &, 40 &, 59 & ,71 & ,73 & ,78 & ,74 & ,75 \\
\hline
\end{tabular}


También se han obtenido valoraciones positivas hacia las condiciones en que los estudiantes realizan sus Grados en Educación ( $M=3,73 ; \mathrm{SD}=, 53)$; prefieren estudiar solos más que de manera grupal, considerándose estudiantes competentes, que muestran interés hacia la actividad académica realizada y que participan activamente en el proceso de estudio. Finalmente, el factor con la puntuación más baja es el relacionado con el nivel de implicación del estudiante hacia el estudio $(M=3,03 ; S D=, 40)$, mostrando su indiferencia hacia cuestiones vinculadas con la asistencia diaria a clase, responsabilidad, autonomía o esfuerzo ante el estudio.

En cuanto a la puesta en práctica de estrategias de trabajo autónomo, el análisis descriptivo desvela cómo la estrategia más utilizada por el estudiante es la vinculada a la preparación de exámenes $(M=3,78 ; S D=, 59)$ seguida de las de conceptualización, es decir, trabajo intelectual sobre el contenido de las materias $(\mathrm{M}=3,66 ; \mathrm{SD}=, 71)$. Las de menor puntuación (con casi un punto de diferencia) se relaciona con la ampliación del material propuesto por el profesor para el estudio de la asignatura $(M=2,89 ; S D=, 75)$, seguida de la colaboración y participación en el desarrollo de tareas grupales $(M=3,06 ; S D=, 74)$ y la puesta en práctica de estrategias de planificación temporal y programación de tareas $(M=3,12 ; S D=, 78)$.

El análisis de correlación para las variables motivacionales, obtenido a partir de coeficiente de correlación de Pearson, determina relaciones estadísticamente significativas en algunas de ellas. El carácter de las relaciones entre variables es moderado en la mayoría de los casos, pues sus valores son superiores a ,30 (Cohen, 1988). Los mejores resultados se relacionan con las variables: metas vitales y los motivos personales del estudiante hacia el aprendizaje $(r=, 60)$, así como sus condiciones de estudio y las estrategias de aprendizaje puestas en práctica $(r=, 53)$. De igual modo, se aprecian relaciones significativas a nivel estadístico entre las variables vinculadas a la puesta en práctica de estrategias de trabajo autónomo, teniendo también carácter moderado en su mayoría (Cohen, 1988). Aquellas en las que se producen mayores correlaciones corresponden a la puesta en práctica de estrategias de preparación de exámenes y trabajo intelectual sobre el contenido de la materia $(r=, 62)$ y planificación de tiempos y programación de tares respectivamente $(r=, 54)$, así como ampliación del material propuesto por el profesor y colaboración en tareas grupales $(r=, 54)$.

Incluso, se aprecian relaciones entre las percepciones de los estudiantes hacia el desarrollo de estrategias de trabajo autónomo y aquellas otras vinculadas a la identificación de la motivación académico-personal hacia los estudios que realizan. Las condiciones hacia el estudio y la implicación del estudiante para la realización de sus estudios universitarios se encuentran positiva y significativamente relacionadas con las variables vinculadas con la preparación de exámenes $(r=, 33)$. De igual modo, se aprecian correlaciones entre la motivación del estudiante para el desarrollo de estrategias de aprendizaje y su implicación en tareas de colaboración ante el proceso de enseñanza-aprendizaje $(r=, 34)$. Las metas vitales del estudiante implicado en la investigación también guardan relaciones significativas a nivel estadístico con la práctica de estrategias de planificación, programación de tareas para el estudio y la realización de trabajos $(r=, 50)$. Finalmente, las actitudes del estudiante hacia el aprendizaje se relacionan significativamente a nivel estadístico con la práctica de estrategias basadas en la preparación de exámenes ( $r=, 38)$, conceptualización de la materia $(r=, 30)$ y planificación y programación de las tareas $(r=, 40)$.

A continuación, el análisis de varianza realizado (ANOVA) ha permitido detectar la existencia de diferencias estadísticamente significativas según la variable sociodemográfica "edad del estudiante" para valores inferiores a ,05. Así pues, la edad del estudiante puede facilitar o, en su defecto, impedir el desarrollo de determinadas estrategias basadas en el aprendizaje autónomo, así como modificar las motivaciones a nivel académico y personal del estudiante para la realización de sus estudios universitarios. Tal y como se muestra en la Tabla 3, y centrados en las estrategias de trabajo autónomo, los resultados obtenidos demuestran diferencias significativas a nivel estadístico para las siguientes variables: estrategias de ampliación $(F(4,186)=3,600 ; \quad p=, 007)$, colaboración $(F(4,183)=2,422 ; \quad p=, 050)$, conceptualización $(F(4,185)=3,384 ; p=, 011)$, preparación de exámenes $(F(4,188)=2,629, p=, 036)$ y participación en el proceso de enseñanza-aprendizaje $(F(4,184)=3,842 ; p=, 005)$. Por su parte, y para la escala sobre autopercepciones del estudiante hacia su motivación académico-personal, existen diferencias a nivel estadístico para la variable vinculada con los motivos personales hacia el aprendizaje $(F(3,184)=3,093 ; p=, 038)$.

La prueba de Tukey realizada a posteriori revela cómo dichas diferencias se sitúan entre los estudiantes más jóvenes (menores de 20 años) y aquellos otros cuya edad supera los 31 años. El análisis de medias sobre las variables ha permitido determinar cómo las valoraciones del alumnado con menos de 20 años se muestran más favorables hacia el desarrollo de estrategias basadas en aprendizaje autónomo, así como los motivos personales que le llevan a realizar dichos estudios universitarios, frente a aquellos otros de mayor edad. Finalmente, el análisis de varianza realizado a partir de la prueba $t$ de Student ha permitido comprobar la significación de diferencias entre grupos, tomando como referencia la variable dicotómica "titularidad de la Universidad en que el estudiante realiza sus estudios de Grado en Educación Infantil o Educación Primaria", pudiendo tener esta un carácter público o privado (Tabla 4). 
Tabla 3: Análisis de varianza ANOVA según la edad del estudiante

\begin{tabular}{|l|c|c|c|c|c|}
\hline & \multicolumn{3}{|c|}{ Edad (M.) } & \multirow{2}{*}{ F. } & \multirow{2}{*}{ Sig. } \\
\cline { 2 - 4 } & $<20$ años & $21-30$ años & $>31$ años & & \\
\hline Motivos personales & $4,38^{*}$ & 3,52 & $3,28^{*}$ & 3,093 &, $038^{*}$ \\
\hline Condiciones estudio & 3,95 & 3,60 & 3,35 &, 834 &, 483 \\
\hline Estrategias aprendizaje & 3,37 & 3,48 & 3,69 &, 316 &, 814 \\
\hline Metas vitales & 3,47 & 3,45 & 2,97 &, 310 &, 818 \\
\hline Actitud aprendizaje & 3,08 & 3,19 & 3,05 & 1,787 &, 165 \\
\hline Preparación & $4,00^{*}$ & 3,91 & $3,69^{*}$ & 2,629 &, $036^{*}$ \\
\hline Conceptualización & $3,82^{*}$ & 3,68 & $3,49^{*}$ & 3,384 &, $011^{*}$ \\
\hline Participación & $4,08^{*}$ & 3,47 & $3,37^{*}$ & 3,842 &, $005^{*}$ \\
\hline Planificación & 3,05 & 3,13 & 3,57 & 1,395 &, 237 \\
\hline Colaboración & $3,93^{*}$ & 3,13 & $2,63^{*}$ & 2,422 &, $050^{*}$ \\
\hline Ampliación & $3,91^{*}$ & 2,85 & $2,82^{*}$ & 3,600 &, $007^{*}$ \\
\hline
\end{tabular}

Los resultados obtenidos confirman la existencia de diferencias significativas a nivel estadístico para algunas de las variables asociadas al desarrollo de estrategias de trabajo autónomo en el estudiante, así como su motivación académica y personal. Más concretamente, las percepciones de los estudiantes resultan ser más favorables para aquellos que pertenecen a la Universidad Católica de Murcia, institución de titularidad privada, en el desarrollo de estrategias basadas en la planificación de sus estudios universitarios $(\mathrm{t}(279)=-1,766 ; \mathrm{p}=, 008)$, colaboración del estudiante en el proceso de enseñanza-aprendizaje $(\mathrm{t}(278)=-9,752 ; \mathrm{p}=, 002)$ y preparación de exámenes $(\mathrm{t}(278)=-8,685 ; \mathrm{p}=, 000)$. Por su parte, y para las variables relacionadas con las autopercepciones de los estudiantes hacia sus motivaciones académico y personales, los resultados revelan valoraciones más favorables hacia los estudiantes de la Universidad de Jaén, institución de titularidad pública. Más concretamente, las diferencias a nivel estadístico se sitúan en las variables relacionadas con las actitudes del estudiante hacia sus estudios universitarios $(t(279)=-7,485$; $\mathrm{p}=, 039)$ y las condiciones e implicación de éste hacia sus estudios en Educación Superior $(\mathrm{t}(279)=-, 9733$; $\mathrm{p}=, 000)$.

Tabla 4: Prueba t de Student para muestras independientes según la titularidad de la Universidad

\begin{tabular}{|l|c|c|c|}
\hline \multirow{2}{*}{} & \multicolumn{2}{|c|}{ Titularidad de la Universidad } & \multirow{2}{*}{ Sig } \\
\cline { 2 - 3 } & Pública & Privada & \\
\hline Motivación & $3,66(, 50)$ & $4,08(, 49)$ & \multirow{2}{*}{, 871} \\
\hline Condiciones estudio & $3,47(, 25)$ & $3,40(, 35)$ & \multirow{2}{*}{, $000^{*}$} \\
\hline Estrategias aprendizaje & $3,05(, 74)$ & $3,46(, 79)$ & \multirow{2}{*}{, 588} \\
\hline Metas vitales & $3,43(, 40)$ & $3,39(, 51)$ & \multirow{2}{*}{, 333} \\
\hline Actitud aprendizaje & $3,03(, 65)$ & $2,95(, 58)$ & \multirow{2}{*}{, $039^{*}$} \\
\hline Preparación exámenes & $2,58(, 47)$ & $3,00(, 40)$ & \multirow{2}{*}{, $000^{*}$} \\
\hline Condiciones implicación estudio & $3,50(, 78)$ & $3,68(, 71)$ &, 358 \\
\hline Participación & $3,69(, 58)$ & $3,45(, 74)$ &, 476 \\
\hline Planificación & $3,28(, 42)$ & $3,55(, 53)$ &, $008^{*}$ \\
\hline Colaboración & $3,11(, 32)$ & $3,43(, 44)$ &, $002^{*}$ \\
\hline Ampliación & $3,09(, 91)$ & $2,88(, 73)$ &, 166 \\
\hline
\end{tabular}

\section{DISCUSIÓN}

Este trabajo ha permitido examinar el perfil del estudiante novel universitario adscrito al Grado en Educación Infantil y Primaria, reflexionando sobre el grado de relación que existe entre sus motivaciones y el desarrollo de estrategias de aprendizaje autónomo que pone en práctica en su proceso formativo. Corresponde al profesorado la obligación de mejorar la calidad de la Educación Superior a partir de la implementación de 
buenas prácticas docentes que garanticen unos mejores resultados académicos (Herrera et al., 2018), y favorezcan la participación y motivación del alumnado hacia sus estudios (De Soto, 2018).

La motivación del estudiante para cursar sus estudios de Grado responde a atribuciones personales hacia el aprendizaje, el cual incluye variables de amistad, aprendizaje, diversión, experiencia universitaria, expectativas personales, familia, futuro, independencia, acceso al mercado de trabajo, profesión, ayudas, responsabilidad, bienestar interior, superación, importancia del saber, vocación, etc. En cuanto a las condiciones de estudio, se destaca su preferencia hacia el trabajo individual, mostrando indiferencia al cuestionar su nivel de implicación hacia el estudio (asistencia a clase, responsabilidad, esfuerzo, autonomía, etc.). Estos resultados justifican la actual preocupación del profesorado universitario por el desarrollo de metodologías participativas que favorezcan el trabajo cooperativo, la capacidad de aprender de manera autónoma y con iniciativa personal, así como su implicación en el proceso formativo (Marcelo y Rijo, 2019). Además, en el estudio desarrollado por Muñoz et al. (2017), se compara el papel de la autoeficacia y la motivación entre estudiantes presenciales y en línea a partir del instrumento utilizado en esta investigación (Boza y Méndez, 2013); se demuestra cómo los estudiantes que provienen de un sistema a distancia tienen mayor conciencia metacognitiva, identifican qué y cómo estudiar, controlan sus estrategias de estudio y se esfuerzan por cumplir los trabajos y tareas de clase.

En cuanto a las estrategias de trabajo autónomo, se destaca su preferencia hacia el desarrollo de habilidades vinculadas a la preparación de exámenes, conceptualización y trabajo intelectual sobre el contenido de las materias incluidas en dichos Grados, así como su participación en el proceso de enseñanza-aprendizaje. Estos resultados muestran una perspectiva tradicional del modelo educativo por parte del estudiante, basado en el almacenamiento de la información y la reproducción de la misma a través de pruebas o exámenes teóricos; desde este punto de vista, lo importante es trabajar el contenido de la materia para preparar el examen y poder aprobar las asignaturas del Grado en que se encuentran. Además, revelan la dificultad del estudiante universitario para emplear las estrategias de trabajo autónomo en su quehacer en el aula universitaria (Başbaği y Yilmaz, 2015), precisando el desarrollo de procesos de capacitación para la competencia "aprender a aprender" que facilite su toma de decisiones respecto a qué y cómo aprender (Gargallo et al., 2020; Trautwein y Bosse, 2017; Wibrowski et al., 2016).

Estos resultados también guardan relación con los obtenidos en otras investigaciones que han hecho uso del "Cuestionario de Estrategias de Trabajo Autónomo" (López-Aguado, 2010) desarrolladas en otros contextos educativos. Así, por ejemplo, Uribe (2012) demuestra cómo los estudiantes de Enfermería de la Universidad de Pamplona (Colombia) desarrollan con frecuencia actividades basadas en el aprendizaje autónomo. Por su parte, y centrados en estudiantes de la carrera de Derecho de la Universidad de Talca (Chile), Riveros y Loyola (2016) determinan cómo las estrategias más desarrolladas por los estudiantes son las de preparación de exámenes y conceptualización, siendo las menos practicadas las de planificación y colaboración entre iguales. Más recientemente, Espinoza-Freire et al. (2017) demostraron cómo las estrategias de aprendizaje autónomo más frecuentemente utilizadas por estudiantes de Educación en una universidad de Ecuador son las referidas al estudio para la evaluación, la participación y asistencia a clase del estudiante, aclaración de dudas, siendo las de menor frecuencia las vinculadas con la implicación del estudiante en tareas grupales y de relación entre iguales. Finalmente, Canova y Pecker (2020) sostienen cómo los estudiantes de Kinesiología y Fisiatría de una institución de Educación Superior de Buenos Aires utilizan con frecuencia estrategias para la preparación de exámenes, llevándose a cabo un bajo uso de las estrategias de planificación.

Ello tiene importantes implicaciones sobre el aprendizaje y el éxito académico del estudiante en Educación Superior. La universidad supone un contexto de aprendizaje, siendo la enseñanza un arte en el que intervienen distintos factores que pueden favorecer o impedir dicho proceso. De este modo, la motivación del estudiante, entendida como su grado de interés para cursar estudios universitarios, le permiten la puesta en práctica de determinadas estrategias de aprendizaje autónomo que posibilitan el desarrollo de la competencia "aprender a aprender".

No obstante, y tras el análisis detallado de los resultados, se demuestra cómo el estudiante universitario lleva a la práctica estrategias de trabajo autónomo de carácter individual (basadas en la preparación de exámenes, conceptualización y trabajo intelectual del contenido), dejando en un segundo plano las estrategias de coordinación y colaboración entre iguales. Ello implica que el estudiante se ayude mutuamente, comparta ideas y desarrolle sus habilidades conjuntamente entre iguales (Maldonado y Sánchez, 2012), maximizando su propio aprendizaje y el de los demás (Johnson y Johnson, 2009). Por tanto, desde la Educación Superior se debe capacitar al estudiante para desarrollar procesos de trabajo colaborativo, siendo necesario trabajar conjuntamente con otros compañeros para crecer y enriquecer nuestra propia vida y la de los demás en todas las competencias. 
Por otra parte, se demuestra cómo los estudiantes de menor edad desarrollan mejores estrategias de aprendizaje autónomo, presentando un perfil más favorable para continuar sus estudios universitarios. Dichos resultados pueden deberse a la apuesta que realiza el actual sistema educativo por mejorar la capacitación de los estudiantes durante la enseñanza obligatoria hacia este tipo de estrategias de aprendizaje, así como el fomento de la inteligencia emocional y el desarrollo de competencias socioemocionales durante su período formativo básico. Estos resultados contradicen las conclusiones expuestas por Tasoobshirazi y Carr (2009), mientras que García-Berbén (2005) o Hernández et al. (2002) demuestran que los alumnos de mayor edad utilizan un enfoque de aprendizaje más profundo. Además, los estudiantes que desarrollan sus estudios de Grado en Educación Infantil o Primaria en la institución de carácter privado muestran mejores estrategias de planificación, colaboración en el proceso de enseñanzaaprendizaje y preparación de exámenes. Por el contrario, aquellos que estudian en la institución de carácter público reconocen estar más motivados para el desarrollo de sus estudios universitarios, así como sus condiciones e implicación en el proceso de enseñanza-aprendizaje. Ello puede estar debido a las condiciones en que se desarrollan el proceso de enseñanza-aprendizaje en ambas instituciones, así como a la formación del profesorado universitario, sus actitudes hacia el aprendizaje de los estudiantes, etc. No obstante, tal y como exponen Maquilón y Hernández (2011), sobre esta circunstancia pueden estar influyendo otra serie de variables.

Por ello, puede resultar de enorme interés realizar una comparativa entre las prácticas docentes desarrolladas por el profesorado universitario de ambas instituciones, al favorecer el desarrollo de estrategias de aprendizaje y motivaciones hacia el estudio entre el alumnado, lo cual repercute de manera directa sobre su rendimiento académico. No obstante, y como posibles limitaciones de la investigación, el uso de cuestionarios como instrumentos de recogida de datos puede generar problemas de deseabilidad social y sinceridad entre los encuestados. Además, dichos instrumentos han permitido analizar valoraciones cuantitativas sobre la muestra objeto de estudio.

\section{CONCLUSIONES}

El análisis de variables motivaciones y de desarrollo de estrategias de trabajo autónomo, así como su grado de relación entre ellas, para estudiantes noveles adscritos al Grado en Educación Infantil y Primaria permite concluir:

1) Las estrategias más habituales de trabajo autónomo del estudiante son las de preparación de exámenes y trabajo intelectual sobre el contenido de las materias que se incluyen en el Grado.

2) El estudiante de Educación accede a dichos Grados por motivos personales, así como por las condiciones en que realizan dichos estudios, preferentemente.

3) Existen relaciones significativas a nivel estadístico entre la motivación del estudiante hacia sus estudios y la puesta en práctica de estrategias de trabajo autónomo.

4) Los estudiantes de menor edad (menos de 20 años) muestran mejores motivos personales para estudiar, a la vez que se muestran más favorables para el desarrollo de estrategias de aprendizaje autónomo (ampliación, colaboración, conceptualización, preparación de exámenes y participación).

5) El estudiante de la universidad privada confirma desarrollar mejores estrategias de planificación, colaboración y preparación de exámenes, mientras que el de la universidad pública considera que dispone de mejores actitudes hacia el estudio y condiciones para realizar el Grado.

\section{REFERENCIAS}

Bandura, A. Self-Efficacy: The exercise of Control, Freeman, New York (1997).

Başbaği, L. y Yilmaz, O. Autonomous foreing language learning, https://doi.org/10.5897/JLC2015.0338, Journal of Languages and Culture, 6(8), 71-79 (2015).

Beishuizen, J. y Steffens, K. A conceptual framework for research on self-regulated learning. Self-regulated Learning in Technology Enhanced Learning Environments Sense Publishers, pp. 3-19. Brill Sense, The Netherlands (2011).

Boza, A. y Méndez, J.M. Aprendizaje motivado en alumnos universitarios: validación y resultados generales de una escala, https://doi.org/10.6018/rie.31.2.163581, Revista de Investigación Educativa, 31(2), 331-347 (2013).

Cabanach, R.G., Valle, A., y tres autores más. Diseño y Validación de un Cuestionario de Gestión Motivacional. Psicodidáctica, ISSN: 1136-1034, 14(1), 29-48 (2009).

Canova, C. y Pecker, L. Características del aprendizaje autónomo en estudiantes de Kinesiología de una institución de Educación Superior de Buenos Aires, https://doi.org/10.11144/Javeriana.ie21-2.caae, Investigación en Enfermería, 21(2), en prensa (2020). 
Castro, E.J. y Miranda, I. Experiencias desmotivacionales y motivacionales de estudiantes varones de Ingeniería para estudiar Matemáticas. El caso de la Universidad Andrés Bello en Santiago de Chile, https://doi.org/10.4067/S071850062019000600083, Formación Universitaria, 12(3), 83-92 (2019).

Castro, M., Zurita, F. y Chacón, R. Motivation towards sports based on sociodemographic variables in university students from Granada. Journal of Sport and Health Research, ISSN: 1989-6239, 11(1), 55-68 (2019).

Cohen, J. Statistical power analysis for the behavioral sciences. $2^{\mathrm{a}}$ Ed., L. Erlbaum Associates, New York (1988).

Coterón, J., Franco, E., Pérez, J. y Sampedro, J. Clima motivacional, competencia percibida, compromiso y ansiedad en Educación Física. Diferencias en función de la obligatoriedad de la enseñanza, Revista de Psicología del Deporte, ISSN: 1132-239X, 22(1), 151-157 (2013).

De Soto, I.S. Herramientas de gamificación para el aprendizaje de ciencias de la tierra, https://doi.org/10.21556/edutec.2018.65.1143, EDUTEC. Revista Electrónica De Tecnología Educativa, 65, 29-39 (2018).

Espinoza-Freire, E., Serrano, O. y Brito, P. El trabajo autónomo en estudiantes de docencia de la Universidad Técnica de Machala, ISSN: 2218-3620, Universidad y Sociedad, 9(2), 202-212 (2017).

Galyon, C.E., Blondin, C.A., y tres autores más. The relationship of academic self-efficacy to class participation and exam performance, https://doi.org/10.1007/s11218-011-9175-x, Social Psychology of Education, 15(2), 233-249 (2012).

García-Berbén, A.B. Estudio de los enfoques de aprendizaje en estudiantes de Magisterio y Psicopedagogía, Electronic Journal of Research in Educational Psychology, ISSN: 1696-2095, 3(2),109-126 (2005).

García-Valcárcel, A. y Tejedor, F.J. Percepción de los estudiantes sobre el valor de las TIC en sus estrategias de aprendizaje y su relación con el rendimiento https://doi.org/10.5944/educXX1.19035, Educación XXI, 20(2), 137-159 (2017).

Gargallo, B. y Suárez, J. Aproximación al perfil de estudiantes universitarios excelentes, Revista de Docencia Universitaria, ISSN: 1887-4592, 12(2), 143-165 (2014).

Gargallo, B., Campos, C. y Almerich, G. Learning to learn at university. The effects of an instrumental subject on learning strategies and academic achievement, https://doi.org/10.1080/11356405.2016.1230293, Cultura y Educación, 28(4), 771-810 (2016).

Gargallo, B., Pérez, C. y tres autores más. La competencia aprender a aprender en la universidad: propuesta de modelo teórico, https://doi.org/10.5944/educXX1.23367, Educación XX1, 23(1), 19-44 (2020).

Garrote, D., Garrote, C. y Jiménez, S. Factores influyentes en motivación y estrategias de aprendizaje de los alumnos de Grado, https://doi.org/10.15366/reice2016.14.2.002, Revista Electrónica Iberoamericana sobre Calidad, Eficacia y Cambio en Educación, 14(2), 31-44 (2016).

Hernández, F., García, M.P. y tres autores más. Consistencia entre motivos y estrategias de aprendizaje en estudiantes universitarios, Revista de investigación educativa, ISSN: 0212-4068, 20(2), 487-510 (2002).

Herrera, L., Souza, M.R. y Soares, J.F. Evaluación de la calidad en la Educación Superior: una revisión de la literatura a partir de la satisfacción del alumnado, https://doi.org/10.18764/2178-2229.v25n2p71-89, Cadernos de pesquisa, 25(2), 71-89 (2018).

Johnson, D.W. y Johnson, R.T. Joining together: group theory and group skills, 10ª Ed., Allyn y Bacon, Boston (2009).

Lee, R.W., Lee, M.J. y Bong, M. Testing interest and self-efficacy as predictors of academic self-regulation and achievement, https://doi.org/10.1016/j.cedpsych.2014.02.002, Contemporary Educational Psychology, 39(2), 86-99 (2014).

López-Aguado, M. Diseño y análisis del Cuestionario de Estrategias de Trabajo Autónomo para estudiantes universitarios, Revista de Psicodidáctica, ISSN: 1136-1034, 15(1), 77-99 (2010).

López-Cárdenas, I., Mella, J. y Cáceres. G. La universidad como ruptura en la trayectoria educativa: experiencias de transición de estudiantes egresados de Enseñanza Media Técnico Profesional que ingresan al Programa Académico de Bachillerato de la Universidad de Chile, https://doi.org/10.4067/S0718-07052018000300271, Estudios Pedagógicos, 44(3), 271-288 (2018).

Lynch, D.J. Confronting challenges: Motivational beliefs and learning strategies in difficult college courses, College Student Journal, ISSN: 0146-3934, 42 (2), 416-421 (2008).

Maldonado, M. y Sánchez, T. Trabajo colaborativo en el aula: experiencias desde la formación docente, Educare, ISSN: 2244-7296, 16(2), 93-118 (2012).

Maquilón, J. y Hernández, F. Identificación de las características del aprendizaje de los estudiantes de Educación Primaria con el cuestionario CEAPS, Anales de Psicología, ISSN: 1695-2294, 27(1), 126-134 (2011).

Marcelo, C. y Rijo, D. Aprendizaje autorregulado de estudiantes universitarios: los usos de las tecnologías digitales, https://doi.org/10.32541/recie.2019.v3i1.pp62-81, Revista Caribeña de Investigación Educativa, 3(1), 62-81 (2019).

Muñoz, S., Sánchez, R., Enríquez, D. y Rosales, C. Contraste de estudiantes universitarios presenciales y en línea en un curso autogestivo: El papel de la autoeficacia y la motivación, https://doi.org/10.21503/hamu.v4i2.1467, Hammut ay, 4(2), 7-16 (2017). 
Nightingale, S.M., Roberts, S. y seis autores más. Trajectories of university adjustment in the United Kingdom: Emotion management and emotional selfefficacy protect against initial por adjustment, https://doi.org/10.1016/j.lindif.2013.08.004, Learning and Individual Differences, 27, 174-181 (2013).

Pegalajar, M.C. Estrategias de trabajo autónomo en estudiantes universitarios noveles de Educación, https://doi.org/10.15366/reice2020.18.3.002, Revista Iberoamericana sobre Calidad, Eficacia y Cambio en Educación, 18(3), en prensa (2020)

Pintrich, P.R. y Schunk, D.H. Motivación en contextos educativos. Teoría, Investigación y Aplicaciones, Pearson, Madrid (2006).

Powell, L., y Robson, F. Learner-Generated Podcasts: A Useful Approach to Assessment?, Innovations in Education and Teaching International, ISSN: 1470-3297, 51(3), 12-21 (2014).

Riveros, C. y Loyola, P. Estudio exploratorio de las características del trabajo autónomo en estudiantes universitarios de la carrera de Derecho, Revista Pedagogía Universitaria y Didáctica del Derecho, ISSN: 0719-5885, 1(3), 89-107 (2016).

Romero, M. y Pérez, M. Como motivar a aprender en la Universidad: una estrategia fundamental contra el fracaso académico en los nuevos modelos educativos, https://doi.org/10.35362/rie5051921, Revista Iberoamericana de Educación, 51, 87-195 (2009).

Roys, J. y Pérez, A. Estrategias de aprendizaje significativo en estudiantes de Educación Superior y su asociación con los logros académicos, Revista Electrónica de Investigación y Docencia, ISSN: 1989-2446, 19, 145-166 (2018).

Simons, B. y Chen, R. Peer and parent influences on school engagement among early adolescents, https://doi.org/10.1177/0044118X09334861, Youth \& Society, 41, 3-25 (2009).

Soares, A.P., Guisande, M.A. y dos autores más. Academic achievement in first-year Portuguese college students: The role of academic Learning to learn at university, https://doi.org/10.1080/00207590701700545, International Journal of Psychology, 44, 204-212 (2009).

Taasoobshirazi, G. y Carr, M. A structural equation model of expertise in college physics, Journal of Educational Psychology, ISSN: 0022-0663, 101(3), 630-643 (2009).

Trautwein, C. y Bosse, E. The first year in higher education-critical requirements from the student perspective, https://doi.org/10.1007/s10734-016-0098-5, Higher Education, 73(3), 371-387 (2017).

Uribe, A. Características del aprendizaje autónomo de los estudiantes del programa de enfermería de la Universidad de Pamplona, Revista Ciencia y Cuidado, ISSN: 1794-9831, 9(1), 24-33 (2012).

Weber, M. y Ruch, W. The role of a good character in 12-year-old school children: Do character strengths matter in the classroom?, https://doi.org/10.1007/s12187-011-9128-0, Child Indicators Research, 5(2), 317-334 (2012).

Wibrowski, C.R., Matthews, W.K. y otro autor más. The Role of a Skills Learning Support Program on First-Generation College Students' Self-Regulation, Motivation, and Academic Achievement: A Longitudinal Study, https://doi.org/10.1177/1521025116629152, Journal of College Student Retention: Research, Theory \& Practice, 19(3), 317-332 (2016).

Yang, M. y Kim, J. Correlation between Digital Literacy and Self- Regulated Learning Skills of Learners in University ELearning Environment Research results, Advanced Science and Technology Lettrers, ISSN: 2287-1233, 71, 80-83 (2014).

Yip, M.C. Learning strategies and self-efficacy as predictors of academic performance: A preliminary study, https://doi.org/10.1080/13538322.2012.667263, Quality in Higher Education, 18, 23-34 (2012).

Zimmerman, B.J., y Schunk, D.H. Handbook of self-regulation of learning and performance, 33-49, Routledge, New York (2011). 
